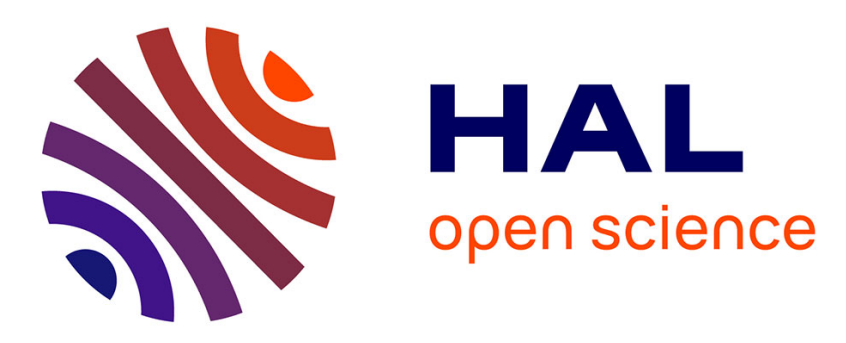

\title{
Underactuated comanipulation for ultrasound breast exams
}

Anja Marx, Marie-Aude Vitrani, Serge Muller, Guillaume Morel

\section{To cite this version:}

Anja Marx, Marie-Aude Vitrani, Serge Muller, Guillaume Morel. Underactuated comanipulation for ultrasound breast exams. IEEE EMBS, Jul 2013, OSAKA, Japan. 10.1109/EMBC.2013.6610639 . hal-01171248

\section{HAL Id: hal-01171248 \\ https://hal.science/hal-01171248}

Submitted on 6 Jul 2015

HAL is a multi-disciplinary open access archive for the deposit and dissemination of scientific research documents, whether they are published or not. The documents may come from teaching and research institutions in France or abroad, or from public or private research centers.
L'archive ouverte pluridisciplinaire HAL, est destinée au dépôt et à la diffusion de documents scientifiques de niveau recherche, publiés ou non, émanant des établissements d'enseignement et de recherche français ou étrangers, des laboratoires publics ou privés. 


\title{
Underactuated Comanipulation for Ultrasound Breast Exams
}

\author{
Anja Marx, Marie-Aude Vitrani, Serge Muller and Guillaume Morel
}

\begin{abstract}
We investigate the possibility of providing adequate task assistance using under-actuated robots for humanrobot tool co-manipulation. This novel approach optimizes robot-user synergy without taking into account any $a$ priori knowledge of parameters depending on the user. Six different actuation modes were compared for a localization and scanning task. The best performance gain was achieved for 1 degree of under-actuation.
\end{abstract}

\section{Comanipulation for Medical Applications}

Co-manipulated devices are systems in which the robot and the user work together to manipulate the same tool. This paradigm is particularly interesting for medical applications, because co-manipulation devices allow the physician to remain with the patient during the intervention. In an ideal co-manipulated system, the medical movement remains unchanged. The robot acts as a device providing active support to improve performance and make the gesture safer.

This paper focuses on co-manipulation systems intended to guide the user by imposing kinematic constraints on the tool.

Schneider et al. propose a semi-passive device called "Passive Arm with Dynamic Constraints" (PADyC, [1]). Its mechanical design allows limiting of tool motions according to a planned task. A geometrical zone is defined in which the surgeon can move freely. When moving out of the zone the surgeon feels forces applied by the robot to move him/her back inside the prescribed zone. This geometrical guidance function is also proposed in [2] and [3]. Davies et al. present a robot for knee surgery named ACROBOT (Active Constraint ROBOT). The co-manipulation does not result from a mechanical constraint but is provided by force control. Different force constraint regions can be defined for the robot. The basic idea behind active constraint control is to gradually increase the stiffness of the robot as it approaches the predefined restricted areas. The same principle is used to control the Surgicobot robot (based on a haptic device) which can be programmed with a desired apparent stiffness within a relatively wide range, but without force sensors [4].

To the best of our knowledge, all systems within this latter class have sufficient actuated degrees of freedom (DOFs) to perform the task without a human user. However, imposing the desired kinematic constraints generally does not require as many actuators. The immediate advantage of actuating less DOFs (i.e. using an under-actuated robot regarding the

This work is partially funded by ANRT under CIFRE grant 247/2009.

A. Marx and S. Muller are with GE Heathcare, Buc, France.

G. Morel, M.-A. Vitrani, and A. Marx are with UPMC Univ Paris 06 , UMR 7222, ISIR, F-75005, Paris, France and CNRS, UMR 7222, ISIR, F-75005, Paris, France task's DOFs) is to have a more compact, less complex system at a lower cost. In this paper, we study possibilities of providing adequate task assistance for co-manipulation tasks by comparing six different robot actuation modes. An initial study has already shown promising results using underactuated guidance [5].

Section II presents the particular medical context of breast cancer detection and the interest in under-actuated co-manipulation. The task is then described and the effects of robot under-actuation on task execution are discussed. Finally, the experimental set-up, protocol and results are dealt with in Section III.

\section{Co-MANipulation for U/S PRobe Guiding}

\section{A. Clinical Context and Medical Gesture}

We applied the principle of co-manipulation for guidance to the medical context of breast cancer diagnosis where the current standard procedure is the succession of an initial mammography (MX) examination and a supplementary Ultrasound (U/S) scan. The surgeon's task is to localize the target lesion defined in the MX images using 2D U/S. The difficulty of this procedure results from the fact that breast geometry changes from one examination to the other due to different patient positions. As in [5], the proposed system facilitates this combined examination by keeping the patient's position, and thus the breast geometry, and by adding a U/S probe guidance robot to the mammography system. The surgeon's task is reduced to $4 \mathrm{DOF}$ w.r.t. the compression paddle: two translations of the probe tip on the paddle (assumed to be planar) one rotation around the normal of the paddle surface, and a second rotation around the intersection line of the paddle surface and the U/S image plane.

\section{B. Task Modelling}

A geometric representation of the task and its set-up can be found in fig.1. The compression paddle is associated with a plane $\pi$. The U/S-plane is denoted by $\mathrm{U}$. The following orthonormal coordinate frames are defined:

- $\mathcal{F}_{P}=\left(P, \vec{x}_{P}, \vec{y}_{P}, \vec{z}_{P}\right)$, the frame attached to the probe $P$ with $\vec{z}_{P}$ being the normal vector of $U$.

- $\mathcal{F}_{0}=\left(O_{0}, \vec{x}_{0}, \vec{y}_{0}, \vec{z}_{0}\right)$, the frame attached to the paddle with $O_{0}$ being a point belonging to the plane $\pi$ and $\vec{z}_{0}$ the plane's normal vector.

Following notions are defined:

- The U/S-probe is handled by the user at $H$.

- The robot impacts on the probe at $R$.

- The mid point of the suspicious lesion is denoted $I$ and $\overrightarrow{I I_{1}}$ denotes its rectangular projection on $U$. 
- The projection of $I$ on the main axis of the U/S-plane $\left(a: \overrightarrow{O_{0} P}+\lambda \vec{y}_{P}=0\right)$ is $\overrightarrow{I I_{2}}$.

Successful accomplishment of the task is defined as the intersection of a and $I$, i.e. when the lesion is centered in the U/S image. The task is hence divided into two parts:

1) lesion intersection with the U/S-plane $\left(I=I_{1}\right)$

2) centering of the lesion in the image $\left(I_{1}=I_{2}\right)$.

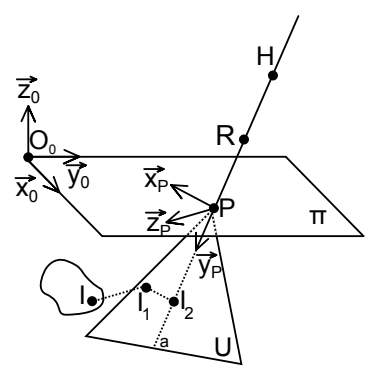

Fig. 1. Geometrical model of a U/S scan through a rigid compression paddle.

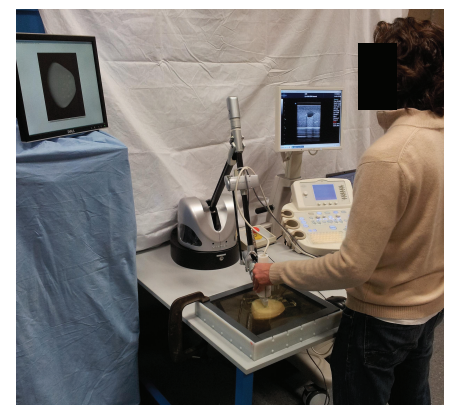

Fig. 2. Subject performing test.

\section{Robot Actuation}

It is important to permit imaging of the surrounding lesion tissues. Consequently, the robot should not completely prevent the user from moving away from the target. To simulate such behavior, a robot control that generates a wrench corresponding to the sum of two compression springs with respective stiffnesses $k_{1}$ and $k_{2}$ was implemented. Both springs have a free length of $0 \mathrm{~mm}$. One connects $I$ and $I_{1}$ whereas the second one connects $I_{1}$ and $I_{2}$ :

$$
\mathcal{E}_{\text {task }}=\left\{\begin{array}{c}
-k_{1} \overrightarrow{I I_{1}} \\
\overrightarrow{0}
\end{array}\right\}_{I_{1}}+\left\{\begin{array}{c}
-k_{2} \overrightarrow{I_{1} I_{2}} \\
\overrightarrow{0}
\end{array}\right\}_{I_{2}}
$$

\section{Underactuation Modes}

In order to take into account the aspect of underaction, $\mathcal{E}_{\text {task }}$ is multiplied with a boolean diagonal matrix A of elements $a_{i}, i \in\{1, \ldots, 6\}$, indicating the under-actuated movements in the probe's frame.

The final wrench applied is hence:

$$
\mathcal{E}_{U}=\left.A \mathcal{E}_{\text {task }}\right|_{P}
$$

There are $2^{6}=64$ possible actuation modes. An a priori analysis of the effect of each possible movement in $\mathcal{F}_{P}$ helps to reduce the number of tested actuation modes to six. Table I sums up the possible forces and moments applicable at $P$ in $\mathcal{F}_{P}$ and their effects on task accomplishment, e.g. centering the lesion $\left(I_{2}=I_{1}\right)$ or assuring intersection between the $\mathrm{U} / \mathrm{S}$-plane and the lesion $\left(I=I_{1}\right)$.

The six compared robot controls are indicated in tab.II. They correspond to a transparent robot (mode 1) and a fully actuated robot (mode 2). Mode 3 only actuates moments, whereas mode 4 actuates only forces. Thus, modes 2 - 4 correspond to standard mechanical systems. In mode 5, two possibilities of helping to establish U/S-plane and lesion intersection $\left(I=I_{1}\right)$ are actuated: forces along $\vec{z}_{P}$ and moments around $\left(P, \vec{x}_{P}\right)$. To complete mode 5 , mode 6 provides additional help to center the lesion in the U/S image $\left(I=I_{1} \wedge I_{1}=I_{2}\right)$ by actuating forces along $\vec{x}_{P}$ and moments around $\left(P, \vec{z}_{P}\right)$.

TABLE I

Underactuation EFfects at P IN $\mathcal{F}_{P}$

\begin{tabular}{lll}
\hline $\begin{array}{l}\text { Under-actuation } \\
\text { components of A }\end{array}$ & $\begin{array}{l}\text { applied force } \\
\text { or moment }\end{array}$ & effect on target-to-U/S-plane relation \\
\hline$a_{1}$ & $\vec{x}_{P}$ & centering $\left(I_{2}=I_{1}\right)$ \\
$a_{2}$ & $\vec{y}_{P}$ & loss of paddle contact \\
$a_{3}$ & $\vec{z}_{P}$ & intersection $\left(I=I_{1}\right)$ \\
$a_{4}$ & $\left(P, \vec{x}_{P}\right)$ & intersection $\left(I=I_{1}\right)$ \\
$a_{5}$ & $\left(P, \vec{y}_{P}\right)$ & intersection $\left(I=I_{1}\right)$ \\
$a_{6}$ & $\left(P, \vec{z}_{P}\right)$ & centering $\left(I_{2}=I_{1}\right)$ \\
\hline
\end{tabular}

TABLE II

ACTUATION MODES COMPARED DURING EXPERIENCES

\begin{tabular}{lcccccc}
\hline Actuated dimensions & $a_{1}$ & $a_{2}$ & $a_{3}$ & $a_{4}$ & $a_{5}$ & $a_{6}$ \\
\hline Mode 1 & 0 & 0 & 0 & 0 & 0 & 0 \\
Mode 2 & 1 & 1 & 1 & 1 & 1 & 1 \\
Mode 3 & 0 & 0 & 0 & 1 & 1 & 1 \\
Mode 4 & 1 & 1 & 1 & 0 & 0 & 0 \\
Mode 5 & 0 & 0 & 1 & 1 & 0 & 0 \\
Mode 6 & 1 & 0 & 1 & 1 & 0 & 1 \\
\hline
\end{tabular}

\section{EXPERIMENTAL VALIDATION}

\section{A. Setup}

Fig.2 depicts the complete test setup. A Virtuose 6D (Haption, France), a highly backdrivable robot was used. Force and torque coordinates can easily be independently set to zero to simulate under-actuation in the robot point of action. These characteristics make it appropriate for testing underactuation modes. An Ultrasonix RP Ultrasound system was used with a 9L linear transducer (Ultrasonix, France). The experimental setup also comprises a phantom box simulating the patient's breast compressed in the mammography system. A computer screen is placed in front of the subject and displays the 3D slice reconstructions of the breast phantom. The subjects are free to navigate within the MX images using the scroll ball of a computer mouse, as it is done during the standard work flow of mammography image reading. MX images were acquired using an investigational 3D MX device based on a Senographe DS (GE Healthcare, Chalfont St Giles, UK). In addition, a controller was implemented to steer the robot.

\section{B. Protocol}

The medical movement of manual U/S breast scans through a compression paddle can be divided into three subtasks. First, by referring only to external breast geometries, the radiologist positions the U/S-probe on the paddle in the approximate position where the target is expected to appear 


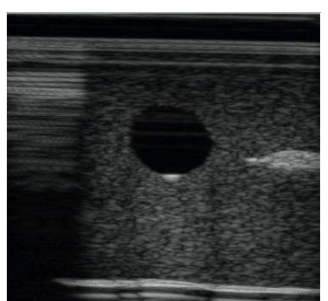

Fig. 3. U/S image during tests. Left: breast border, Top: artifacts due to compression paddle, Bottom: artifacts due to plate simulating the detector, Middle: cystic lesion (black) and tumor lesion (white)

in the U/S images. If the U/S image does not show a lesion, the probe is moved until a first guess at the lesion localization is obtained.

In a second consecutive step, the radiologist images the target's surrounding tissue to verify the proper target localization, notably its position w.r.t. other points in the breast. Once the radiologist is assured about the proper lesion localization, subtask 3 consists of a precise scan of the lesion made to determine its nature (e.g. contours, size). To compare the different performances, a rest probe position was determined in one corner of the breast phantom.

The two tests performed are:

- test 1: subtask one and two: approximate target localization followed by proper target identification.

- test 2: subtask three: entire target lesion-scan.

Four different scanning methods were compared with test two, while all were executed with six different robot control (i.e. a total of 24 runs for test two per subject). First, the user was asked to visualize the lesion in the U/S images under as many different perspectives as possible within 30sec. For the three remaining scanning methods, the subjects were asked to execute a particular movement and had only $10 \mathrm{sec}$ to scan the lesion: translations on the compression paddle, rotations around the paddle's normal vector $\vec{z}_{0}$ and rotations around the intersection of the U/S-plane and the paddle surface $\left(\vec{z}_{P} \wedge\right.$ $\left.\vec{z}_{0}\right)$.

To avoid increasing learning effects, all tests 1 have been executed in a row and prior to tests 2 . The subjects have thus not been accustomed to the phantom during tests one. A total of 22 subjects performed all test series in a randomized fashion (fig.2).

\section{Results and Discussion}

1) Test 1: Users performed worst regarding precision with mode 1, i.e. with a transparent robot (fig.4). In this mode, the mean U/S-plane to target distance was the largest with $0.8 \mathrm{~cm}(\sigma=1.43 \mathrm{~cm})$. As expected, users performed best in terms of precision with a fully actuated robot, mode 2 . The mean distance was only $0.05 \mathrm{~cm}(0.17 \mathrm{~cm})$, which represents an improvement of $93.58 \%$ w.r.t. mode 1. Using modes 3 and 4 , subjects achieved a precision increase of $62.88 \%$ and $66.37 \%$ respectively. The measured mean U/S-plane to target distances were $0.31 \mathrm{~cm}(0.45 \mathrm{~cm})$ and $0.28 \mathrm{~cm}(1.18 \mathrm{~cm})$ repectively.
Results of for modes 5 and 6 are were better than for modes 3 and 4, however there they were not as hight good as those for mode $1: 0.17 \mathrm{~cm}(0.79 \mathrm{~cm})$ and $0.12 \mathrm{~cm}(0.41 \mathrm{~cm})$ mean distance for mode 5 and 6 respectively. These results show a $79.69 \%$ and $85.07 \%$ respective improvement in terms of precision.

The time needed to localize the lesion was one of the longest in mode 1 , taking up $20.11 \%(10.58 \%)$ of the entire time span for tests 1 . Only mode 3 was slower with $21.61 \%$ $(8.61 \%)$, which decreased the test duration by $7.47 \%$. In modes 2, 4 and 6, localization time was similar $(14.26 \%$ $(6.53 \%), 13.55 \%(7.01 \%)$ and $13.71 \%(9.28 \%))$. The The duration reduction percentages are $29.10 \%, 32.59 \%$ and $31.79 \%$ respectively for those three modes. Mode 5 still reached $16.66 \%$ improvement w.r.t. mode 1, i.e. localization time was $16.76 \%(9.84 \%)$.

One factor ANOVA, with subjects as repeated measures, was run for each indicator. The 6 actuation modes have a significant effect on the U/S-plane to target distances $\left(F_{5}=3.983, \mathrm{p}=0.0023902\right)$. Results of completion time $\left(F_{5}=2.9363, \mathrm{p}=0.016029\right)$ showed a less but still significant effect for the 6 experimental conditions. Both conclusions are valid to a significance level of $5 \%$.

2) Test 2: With mode 1 and 3, scanning time was $56.74 \%$ $(3.33 \%)$ and $58.84 \%(6.57 \%)$ of the total time (fig.5). Users thus only spent about half of the time imaging the lesion, whereas it was not visible in the U/S images. However with mode 2 and 4 to 6 , scanning time was between $89.75 \%$ $(3.36 \%)$ and $94.74 \%(2.42 \%)$, which is equivalent to an improvement percentage of $58.18 \%$ to $66.97 \%$ w.r.t. mode 1.

The maximum U/S-plane to target distance is the largest in modes 1 and $3(0.95 \mathrm{~cm}(0.47 \mathrm{~cm})$ and $0.89 \mathrm{~cm}(0.38 \mathrm{~cm}))$, compared to distances between $0.12 \mathrm{~cm}(0.05 \mathrm{~cm})$ and $0.19 \mathrm{~cm}$ $(0.15 \mathrm{~cm})$ for modes 2 and 4 to 6 . The maximum distance was thus decreased by $79.61 \%$ to $87.41 \%$ in modes 2 and 4 to 6 . A similar relation can be observed for the mean U/S-plane to target distance. Mean distance values are $0.35 \mathrm{~cm}(0.09 \mathrm{~cm})$ and $0.36 \mathrm{~cm}(0.11 \mathrm{~cm})$ for modes 1 and 3 respectively. With modes 2 and 4 to 6 , only between $0.05 \mathrm{~cm}(0.01 \mathrm{~cm})$ and $0.07 \mathrm{~cm}(0.05 \mathrm{~cm})$ mean U/S-plane to target distance was measured, corresponding to improvements between $79.76 \%$ and $86.41 \%$.

One factor ANOVA run with subjects as repeated measures again showed a significant effect of the six actuation modes: $F_{5}=25.2254, \mathrm{p}<0.0001$ for scanning time, $F_{5}=18.7332, \mathrm{p}<0.0001$ for $\max U / S-p l a n e$ to target distance and $F_{5}=23.4730, \mathrm{p}<0.0001$ for mean U/S-plane to target distance. P-values are equally below $10^{-4}$ for results of each independent test 2 series (i.e. free movement of all three imposed movements).

Contrary to what was expected, one can not state a significant difference between the scanned target volumes for each mode $\left(F_{5}=1.5832, \mathrm{p}=0.22456\right)$. Even if the users spend more time scanning the lesion in modes 2 and 4 to 6 , they do not take advantage of this to explore the entire lesion. They image the same parts repetitively. The only statistically 
significant result regarding the scanned volume $\left(F_{5}=9.0250\right.$, $\mathrm{p}<0.0001)$ was obtained for an imposed translational movement. $87.54 \%$ and $88.50 \%$ scanned volume for modes 1 and 3 , contrary to $71.99 \%$ to $73.45 \%$ for modes 2 and 4 to 6 . This is probably due to the fact that the user made uncontrolled movements in modes 1 and 3. Subjects reported disliking mode 3 (where only moments were applied) because the robot 'turned the probe in an unusual manner'.
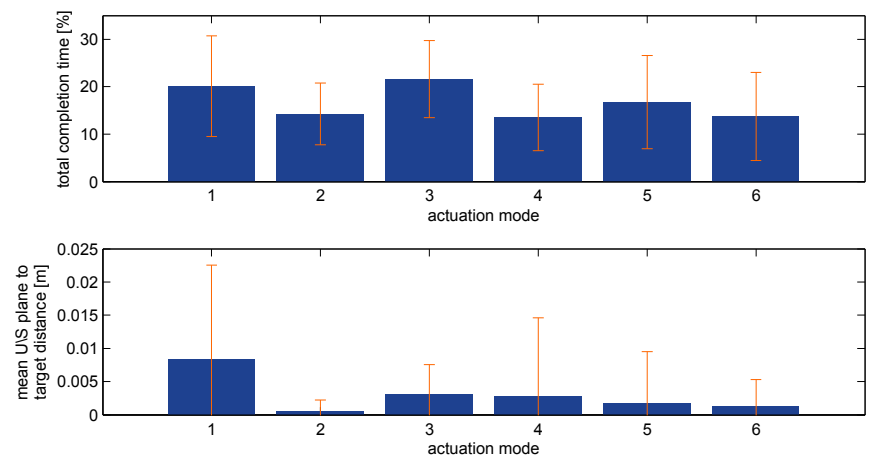

Fig. 4. Results of tests 1: mean localization time and U/S-plane to target distance.
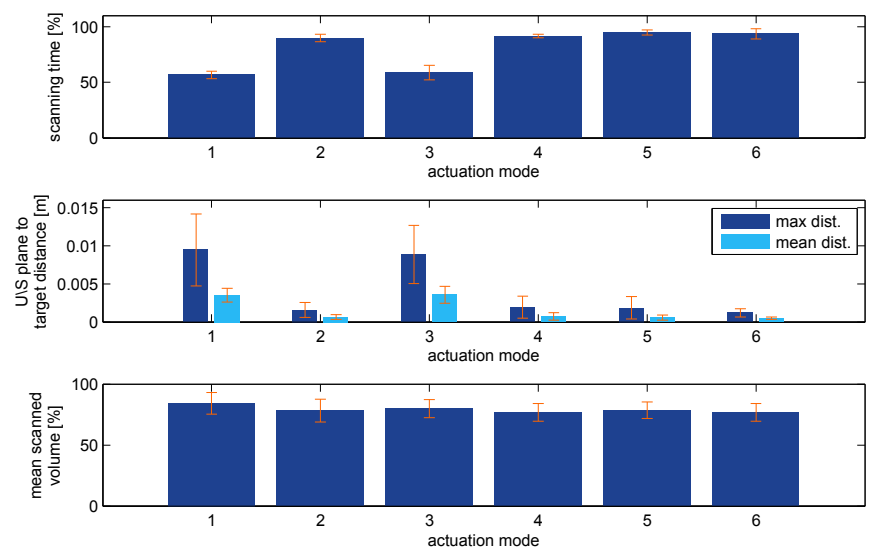

Fig. 5. Results of tests 2: merged results of scanning time, U/S-plane to target distance and scanned volume.

3) Discussion: One can clearly identify mode 6 as the best performing mode with a $13.30 \%$ improvement w.r.t. mode 1 , followed by mode 2 with a $10.77 \%$ improvement. This is certainly a surprising result since mode 6 is underactuated. However, modes 2 and 6 are very close in terms of robot control. Mode 6 only leaves out moments around $\vec{y}_{P}$. Rotations around $\vec{y}_{P}$ help intersection with the U/S-plane and the target. This movement is overloaded twice in mode 6.

From a conceptual point of view, modes 5 and 6 do not correspond to a standard mechanical system. This would mean elevated manufacturing costs which may not justify their slight performance advance compared to common mechanisms as represented by mode 4. Mode 4, applying only forces, increased system precision by $66.37 \%$ and reduced completion time by $32.59 \%$ during tests 1 . Performances for test 2 in mode 4 are comparable to modes 5 and
6 , reaching $91.57 \%$ scanning time and about $80 \%$ precision gain. Mode 4 may therefore be a compromise bewteen performance enhancement and reduction of manufacturing costs.

Users jointly rejected mode 3 due to the uncommon haptic sensations it provokes when the U/S-probe is turned in the user's hand (remember that mode 3 only actuates rotations). This is shown by its poor performances w.r.t. mode 1, especially for localization time in test 1 , where test duration even decreased by $7.47 \%$. For test 2 , mode 3 did not show any overall improvement. It is however interesting to notice, that users were capable of increasing precision during test 1 $(62.88 \%)$ despite this 'unhandy' mode 3.

\section{CONCLUSIONS}

Different modes for adequate task assistance using underactuated robots for tool guidance were compared. The example studied focuses on U/S examinations consecutive to $3 \mathrm{D}$ MX scans for early breast cancer detection.

In this approach, a robot co-manipulates a hand-held U/Sprobe and is programmed to assist the user in localizing and scanning a lesion previously identified thanks to a mammography scan. It provides force feedback to help establish a U/S-plane and target intersection as well as centering the lesion in the U/S image. This guidance is provided by applying simple forces and torques on the U/S probe. Depending on the simulated degree of under-actuation, force and moment components can be set to zero to simulate underactuation of the robot.

User performances using different under-actuation modes of the system to conduct a scanning task were compared to data on usual U/S scanning without active robot support. Four under-actuation modes were tested. Performance gains for localization/pointing tasks can be achieved using any of the under-actuated modes other than mode 3 . The best underactuated guidance was provided with only 1 degree of underactuation, compared to the 5DOF of the task in $\mathcal{F}_{P}$. It is thus possible to obtain synergistic robot-human behavior using a partial-guidance system. The missing actuated DOF are compensated for the user knowing the actual task to perform.

\section{REFERENCES}

[1] O. Schneider, J. Troccaz, O. Chavanon, and D. Blin, "Padyc: a synergistic robot for cardiac puncturing," in Proc. IEEE International Conference on Robotics and Automation(ICRA'00), Apr. 2000, pp. 2883-2888.

[2] B. Davies, K. Fan, R. Hibberd, M. Jakopec, and S. Harris, "A mechatronic based robotic system for knee surgery," in Proc. IASTED International Conference on Intelligent Information Systems (IIS'97), Dec. 1997, pp. 48-52.

[3] E. Bonneau, F. Taha, P. Gravez, and S. Lamy, "Surgicobot: Surgical gesture assistance cobot for maxillo-facial interventions," Perspective in Image-Guided Surgery, pp. 353-360, Mar. 2004.

[4] V. Francoise, A. Sahbani, and G. Morel, "A comanipulation device for orthopedic surgery that generates geometrical constraints with real-time registration on moving bones," in Robotics and Biomimetics (ROBIO), 2011 IEEE International Conference on, dec. 2011, pp. 38 -43.

[5] A. Marx, M.-A. Vitrani, B. Herman, R. Iordache, S. Muller, and G. Morel, "Synergic comanipulation despite underactuated robot," in Robotics and Automation (ICRA), 2011 IEEE International Conference on, may 2011, pp. $5756-5761$. 